\title{
OPTIMAL CONTROL FOR SWITCHED DISTRIBUTED PARAMETER SYSTEMS WITH APPLICATION TO THE GUIDANCE OF A MOVING ACTUATOR
}

\author{
Orest V. Iftime ${ }^{* 1}$ Michael A. Demetriou ${ }^{* *}$ \\ * Delft Center for Systems and Control, Mekelweg 2, 2628 \\ CD, Delft, The Netherlands, o.v.iftime@dcsc.tudelft.nl \\ ** Dept of Mech. Eng., Worcester Polytechnic Institute, \\ Worcester, MA01609-2280,USA, mdemetri@wpi.edu
}

\begin{abstract}
The present study considers a hybrid controller scheme for the optimal switching of a moving (or scanning) actuator (MA) for a class of distributed parameter systems (DPS). The proposed hybrid controller switches both the location and control signal of the actuator at the beginning of a time interval and remains unchanged over the duration of the time-interval. As time progresses, the above policy is repeated over a sequence of "time windows", thus generating an algorithmic procedure which results in the actuator/controller switching policy. Guiding the MA at certain a priori selected actuator positions at different timeintervals is made possible by solving a double optimization problem. The decision method for actuator switching utilizes a finite-horizon LQR optimal control policy and, using optimality criteria, the associated optimal control problem is solved backwards-in-time for several time subintervals. A numerical example with simulation results is presented. Copyright ${ }^{\complement}$ 2005 IFAC.
\end{abstract}

Keywords: Moving actuator (MA), actuator scheduling, hybrid systems, distributed parameter systems (DPS), optimal control, switching algorithms.

\section{INTRODUCTION}

We consider a class of hybrid DPS in which both the location of the actuating device and the associated control signal are allowed to switch at different time intervals. The motivation for this actuator/controller switching stems from the desire to enhance performance. As was already observed in thermal manufacturing applications (Demetriou et al., 2003) a significant improvement is observed when an actuating device is allowed to move at preselected positions in the spatial domain. Unlike the finite dimensional case of hy-

\footnotetext{
1 University of Groningen, Faculty of Economics, Landleven 59747 AD, Groningen, The Netherlands
}

brid systems where only the controller signals are switched (Borrelli, 2003; Liberzon, 2003; Savkin and Evans, 2002), the case of distributed parameter systems incorporates information on the spatial domain and thus allows also for the switching of the actuator positions.

The problem under study is formulated in the next section. Results on the finite horizon optimal control are presented in Section 3 and the algorithm for the optimal actuator switching policy for a family of switched distributed parameter systems is provided. In Section 4, a switching policy of MA on infinite-time interval is considered. Using a finite number of switches or global stability, an optimal and an $\epsilon$-optimal algorithm, respectively, 
is proposed. An example of a heat equation system described by a parabolic partial differential equation along with its numerical results are presented in Section 5. Conclusions follow in Section 6.

\section{PROBLEM FORMULATION}

Let $\left(S_{p}\right)_{p \in \mathcal{P}}$, for some index set $\mathcal{P}$, be the family of linear continuous-time systems which, for each fixed $p \in \mathcal{P}$, is given by a state linear system $\left(A, B_{p}, C_{p}\right)$ of the form

$$
\left(S_{p}\right)\left\{\begin{array}{l}
\frac{d}{d t} z(t)=A z(t)+B_{p} u(t), \\
y(t)=C_{p} z(t)
\end{array}\right.
$$

where the operator $A$ is the infinitesimal generator of a strongly continuous semigroup $T(t)$ on a Hilbert space $Z$, and $B_{p}$ and $C_{p}$ are bounded linear operators from a Hilbert space $U$ to $Z$, and from $Z$ to a Hilbert space $Y$, respectively. To $\left(S_{p}\right)_{p \in \mathcal{P}}$ is associated the set of all possible switches between the given systems

$$
\Sigma=\left\{\sigma \mid \sigma:\left[t_{0}, \infty\right) \rightarrow \mathcal{P}, \text { piecewise constant }\right\} .
$$

The family of switched systems $\left(\left(S_{p}\right)_{p \in \mathcal{P}}, \Sigma\right)$ taken under consideration in this paper are the hybrid dynamical systems consisting of the family of continuous-time systems $\left(S_{p}\right)_{p \in \mathcal{P}}$ together with all switching rules $\sigma \in \Sigma$, all initial states $z\left(t_{0}\right)=$ $z_{0} \in Z$, and all inputs $u \in L_{2}\left(\left[t_{0}, \infty\right) ; U\right)$. Denote by $t_{0}<t_{1}<t_{2}<\ldots<t_{n}<\ldots$ the countable set of switching time instants, namely the discontinuities of the switching functions $\sigma$. For simplicity, we consider here only equidistant switches, i.e., for a given $\Delta t$, consider $t_{i}:=t_{0}+i \Delta t, i \in \mathbb{N}$.

Suppose that a switching function $\sigma$, an initial condition $z_{0}$ and an input $u$ are given. Then, on each interval $\left\{\left[t_{i}, t_{i+1}\right)\right\}_{i \in \mathbb{N}}$, the state $z_{\sigma}(t)$ of the switched system $\left(\left(S_{p}\right)_{p \in \mathcal{P}}, \sigma\right)$ is the mild solution of the Cauchy problem (1) (see (Curtain and Zwart, 1995, Chapter 3)), i.e. for $t_{i} \leq t \leq t_{i+1}$

$$
z_{\sigma}(t)=T(t) z_{\sigma}\left(t_{i}\right)+\int_{t_{i}}^{t} T(t-s) B_{\sigma\left(t_{i}\right)} u(s) d s .
$$

Then, the output trajectory on each interval $\left\{\left[t_{i}, t_{i+1}\right)\right\}_{i \in \mathbb{N}}$ is

$$
y_{\sigma}(t)=C_{\sigma\left(t_{i}\right)} z_{\sigma}(t) .
$$

The initial conditions at the beginning of each interval are $\left\{z_{\sigma}\left(t_{i}\right)\right\}_{i=0}^{n-1}$, and they are considered to be the end values of the solution on the preceding time-interval. Consequently, the mild solution of any of the switched system $\left(\left(S_{p}\right)_{p \in \mathcal{P}}, \sigma\right)$ is continuous. With the trajectories (2),(3) we associate the following cost functional

$$
\begin{aligned}
& J_{\sigma}\left(z_{0} ; u, t_{0}, t_{f}\right)=\left\langle z_{\sigma}\left(t_{f}\right), M z_{\sigma}\left(t_{f}\right)\right\rangle \\
& \quad+\int_{t_{0}}^{t_{f}}\left(\left\langle y_{\sigma}(s), y_{\sigma}(s)\right\rangle+\langle u(s), R u(s)\rangle\right) d s,
\end{aligned}
$$

where $z_{0} \in Z$ is the initial condition, $u \in$ $L_{2}\left(\left[t_{0}, t_{f}\right] ; U\right)$ is the input trajectory, $M$ is a selfadjoint, nonnegative, bounded operator on $Z$ and $R$ is a coercive, bounded operator on $U$. The final time can be finite, $t_{f}<\infty$, or infinite, $t_{f}=\infty$.

The control objective is to minimize the cost (4) over all possible trajectories (2),(3) of the switched systems from $\left(\left(S_{p}\right)_{p \in \mathcal{P}}, \Sigma\right)$. One may then formulate the following double optimization problem.

Problem 1. Given a family of switched systems $\left(\left(S_{p}\right)_{p \in \mathcal{P}}, \Sigma\right)$, and an initial condition $z_{0} \in Z$, find an optimal control $u_{o p t} \in L_{2}\left(\left[t_{0}, t_{f}\right] ; U\right)$ and an optimal switching function $\sigma_{o p t} \in \Sigma$ that minimize the cost functional (4) over all possible trajectories (2),(3). In other words, find

$$
J_{o p t}=\min _{\sigma \in \Sigma,} \min _{u \in L_{2}\left(\left[t_{0}, t_{f}\right], U\right)} J_{\sigma}\left(z_{0} ; u\right) .
$$

Motivated by engineering applications and in particular by thermal manufacturing (Demetriou et al., 2003), we introduce additional assumptions. In thermal manufacturing applications, the actuator device (heat source) will most likely be mounted on a robotic arm, and will not transverse large distances in infinitesimal time intervals. In addition, due to computational restrictions, online optimization at every time instance is not feasible. Instead, it is assumed that the moving actuator can reside at a given location for a time interval of small duration (dwell time) whose length is dictated by hardware limitations and bandwidth, and closed loop system stability bounds. Thus, we will look at the optimal problem at each of the subintervals. As mentioned earlier, we will consider time intervals of constant length.

Assumption 2. (Finite candidate locations). There are only a finite number $m \geq 2$ of admissible locations for the moving actuator. Denote by $\mathcal{P}^{m}=$ $\left\{p_{1}, p_{2}, \ldots, p_{m}\right\} \subset \mathcal{P}$ the corresponding index set, and by $\Sigma^{m}$ the set of switching functions $\sigma$.

Assumption 3. (Zero transverse time). The time required by the actuating device to transverse from location $p_{1} \in \mathcal{P}$ at the end of the time interval $\left[t_{i}, t_{i}+\Delta t\right]$ to location $p_{2} \in \mathcal{P}$ for the subsequent interval $\left[t_{i}+\Delta t, t_{i}+2 \Delta t\right]$ is negligible and may be assumed to be zero, i.e. one has inertialess moving source. Alternatively, one may assume that there are many actuators available, and only one is to be activated and stay active throughout a given time interval. Thus the actuator at location 
$p_{1} \in \mathcal{P}$ will be active during the time interval $\left[t_{i}, t_{i}+\Delta t\right]$ and the actuator at location $p_{2} \in \mathcal{P}$ will be activated for the subsequent interval $\left[t_{i}+\right.$ $\left.\Delta t, t_{i}+2 \Delta t\right]$ with no additional time required for activating and disengaging $p_{2}$ and $p_{1}$, respectively.

Assumption 4. (Minimum residence time). The choice of the residence time $\Delta t$ is chosen to be larger than the minimum dwell time $\tau_{d}$ (Liberzon, 2003), allowable for stability under switching.

Continuing, one may now formulate the following two optimization problems (the final time can be finite, $t_{f}<\infty$, or infinite, $\left.t_{f}=\infty\right)$.

Problem 5. Given a family of switched systems $\left(\left(S_{p}\right)_{p \in \mathcal{P}^{m}}, \Sigma^{m}\right)$ which satisfies Assumptions 3 and 4 , and an initial condition $z_{0} \in Z$, find an optimal control $u_{\text {opt }} \in L_{2}\left(\left[t_{0}, t_{f}\right] ; U\right)$ and an optimal switching function $\sigma_{o p t} \in \Sigma^{m}$ that minimize the cost functional $J_{\sigma}\left(z_{0} ; u, t_{0}, t_{f}\right)$ over all possible trajectories $(2),(3)$, i.e. solve

$$
J_{o p t}^{m}\left(z_{0} ; t_{0}, t_{f}\right)=\min _{\sigma \in \Sigma^{m}, u \in L_{2}} J_{\sigma}^{m}\left(z_{0} ; u, t_{0}, t_{f}\right) .
$$

Problem 5 has two degrees of freedom. We seek an optimal control from a class of available controls. Please note that Problem 5 can be found in the general classification provided in (Butkovskiy and Pustyl'Nikov, 1987, Figure 1.4, page 45).

The above formulations of the optimization problems for switched systems are more general than one needs for providing methodologies for optimal and suboptimal efficient switching of a moving actuator for distributed parameter systems. The generality comes from the fact that, for the set of systems (1), we have not imposed the restriction that the bounded operators $\left(B_{p}\right)_{p \in \mathcal{P}}$ model point actuators and sensors. Usually, a point actuator and/or sensor is modelled as a delta distribution in the point where the actuator is applied, which does not "always" represent a bounded operator. This is one way to view the problem and provide an optimal trajectory for the actuator position, as treated in (Butkovskiy and Pustyl'Nikov, 1987).

\section{OPTIMAL SWITCHING POLICY OF MA ON FINITE HORIZON}

In this section we consider the final time $t_{f}<\infty$. Assume that $\left(\left(S_{p}\right)_{p \in \mathcal{P}^{m}}, \Sigma^{m}\right)$ satisfies Assumptions 3 and 4 and an initial condition $z_{0} \in Z$ is given. An algorithm for solving the formulated optimal control problem (Problem 5) on a finitetime interval $\left[t_{0}, t_{f}\right]$ is provided. We must first consider a set of $m$ fixed values (locations) $\mathcal{P}^{m}=$ $\left\{p_{1}, p_{2}, \ldots, p_{m}\right\}$ in which the switching function may take values (the MA may reside at these locations during certain time intervals).

We briefly introduce the notations and summarize well-known results from optimal control without switching, i.e. $\sigma(t)=p$ for all $t \geq 0$, (Curtain and Zwart, 1995). For each fixed actuator location $p \in \mathcal{P}^{m}$, the optimal control signal that minimizes a finite horizon cost of the type (4) on the finite time interval $\left[t_{a}, t_{b}\right]$ over all trajectories of the system $S_{p}, z_{0}=z\left(t_{a}\right)$, is given by

$$
u_{p}^{o p t}\left(t ; z_{0}, t_{a}, t_{b}\right)=K_{p}(t) z_{p}^{o p t}\left(t ; z_{0}, t_{a}, t_{b}\right),
$$

with $K_{p}(t) \triangleq-R^{-1} B_{p}^{*} \Pi_{p}(t)$. The optimal state, $z_{p}^{o p t}\left(t ; z_{0}, t_{a}, t_{b}\right)$, is the mild solution of the abstract evolution equation

$$
\begin{aligned}
& \frac{d}{d t} z(t)=\left(A-B_{p} K_{p}(t)\right) z(t) \triangleq A_{c l, p}(t) z(t) \\
& z\left(t_{a}\right)=z_{0} .
\end{aligned}
$$

The self-adjoint nonnegative operator $\Pi_{p}(t) \in$ $\mathcal{L}(Z)$ for all $t \in\left[t_{a}, t_{b}\right]$, and satisfies the Operator Differential Riccati Equation (ODRE)

$$
\begin{aligned}
& \frac{d}{d t}\left\langle\phi, \Pi_{p}(t) \psi\right\rangle=-\left\langle\phi, \Pi_{p}(t) A \psi\right\rangle \\
& \quad-\left\langle A \phi, \Pi_{p}(t) \psi\right\rangle-\left\langle C_{p} \phi, C_{p} \psi\right\rangle \\
& \quad+\left\langle\Pi_{p}(t) B_{p} R^{-1} B_{p}^{*} \Pi_{p}(t) \phi, \psi\right\rangle, \quad t \in\left[t_{a}, t_{b}\right], \\
& \Pi_{p}\left(t_{b}\right)=M_{p},
\end{aligned}
$$

for $\phi, \psi \in \mathcal{D}(A)$. The following relationship between the minimum of $J_{p}\left(z_{0} ; t_{a}, t_{b}, u\right)$, defined by (7), and $\Pi_{p}\left(t_{a}\right)$ holds

$$
\min _{u \in L_{2}\left(\left[t_{a}, t_{b}\right], U\right)} J_{p}\left(z_{0} ; t_{a}, t_{b}, u\right)=\left\langle z_{0}, \Pi_{p}\left(t_{a}\right) z_{0}\right\rangle .
$$

The following algorithm provides a solution for Problem 5.

Algorithm 1. Consider a family of switched systems $\left(\left(S_{p}\right)_{p \in \mathcal{P}^{m}}, \Sigma^{m}\right)$.

Part A Solving $m$ ODREs in each subinterval backwards in time with the terminal condition for an interval being the initial condition from the next subinterval.

Step 1: Divide the interval $\left[t_{0}, t_{f}\right]$ into $k=\left[\frac{t_{f}-t_{0}}{\Delta t}\right]$ sub-intervals of length $\Delta t$. ([·] denotes the integer part of a real number).

Step 2: If $t_{f}>k \Delta t$ solve ODRE (9) with the initial condition $\Pi_{\sigma(k \Delta t)}\left(t_{f}\right)=M$, on the interval $\left[k \Delta t, t_{f}\right]$, for every possible value of $\sigma(k \Delta t)$. Set $M_{\sigma(k \Delta t)}=\Pi_{\sigma(k \Delta t)}(k \Delta t)$. Else, for each $\sigma(k \Delta t)=$ $p \in \mathcal{P}^{m}$, set $M_{\sigma(k \Delta t)}=M$ and proceed with Step 3.

Step 3: Solve, on the interval $\left[t_{k-1}, t_{k}\right]$, the ODRE (9) for each of the $m$-initial conditions $\Pi_{\sigma\left(t_{k-1}\right)}\left(t_{k}\right)=M_{\sigma\left(t_{k}\right)}$ and each value of $\sigma\left(t_{k-1}\right)$. 
Step 4: Continue the procedure until the last interval $\left[t_{0}, t_{1}\right]$.

Part B Finding the optimal switching $\sigma^{o p t}$ and $u_{\sigma^{o p t} \text {. }}^{\text {opt }}$.

Step 5: Find the minimal cost of (6) as

$$
\begin{aligned}
J_{o p t}^{m}\left(t_{0}, t_{f}\right) & =\min _{\sigma \in \Sigma^{m}} J_{\sigma}^{m}\left(z_{0} ; t_{0}, t_{f}, u_{\sigma}^{o p t}\right) \\
& =\min _{\sigma \in \Sigma^{m}}\left\langle z_{0}, \Pi_{\sigma}\left(t_{0}\right) z_{0}\right\rangle .
\end{aligned}
$$

Step 6: Choose $\sigma^{\text {opt }}$ as the switching function corresponding to the optimal cost $J_{o p t}^{m}\left(t_{0}, t_{f}\right)$. Then the optimal input is $u_{\sigma^{o p t}}^{o p t}$.

The total number of ODRE needed to be solved in the above algorithm can easily be computed; e.g. for $t_{f}=k \Delta t$ one has to solve $\sum_{j=1}^{k} m^{k}$ ODREs.

Theorem 6. Consider the switched system $\left(\left(S_{p}\right)_{p \in \mathcal{P}}, \sigma\right)$ with the cost functional (4). For every $z_{0} \in Z$ there exists a unique input $u_{\sigma}^{\text {opt }}\left(\cdot ; z_{0}, t_{0}, t_{f}\right) \in L_{2}\left(\left[t_{0}, t_{f}\right], U\right)$ such that

$$
J\left(z_{0} ; t_{0}, t_{f}, u_{\sigma}^{o p t}\left(\cdot ; z_{0}, t_{0}, t_{f}\right)\right) \leq J\left(z_{0} ; t_{0}, t_{f}, u\right)
$$

for all $u \in L_{2}\left(\left[t_{0}, t_{f}\right], U\right)$. Moreover, the Algorithm 1 is optimal.

Simple properties that can be associated to the above analysis are stated in the following lemma.

Lemma \%. Consider a fixed switching function $\sigma \in \Sigma^{m}$. Then the following statements hold:

(1) The optimal trajectory does not depend on the initial choice of the time $t_{0}$.

(2) If $t_{0} \leq t_{1} \leq t_{2} \leq t_{k}$, then $\Pi_{\sigma}\left(t_{2}\right) \leq \Pi_{\sigma}\left(t_{1}\right)$.

(3) $\Pi_{\sigma}(\cdot)$ is strongly continuous from the right in $\left[t_{0}, t_{f}\right]$.

(4) If $t_{0} \leq t_{1} \leq t_{2}$, then

$$
J_{\sigma}^{m}\left(z_{0} ; u_{\sigma}^{o p t}, t_{0}, t_{1}\right) \leq J_{\sigma}^{m}\left(z_{0} ; u_{\sigma}^{o p t}, t_{0}, t_{2}\right) .
$$

Using relations between costs on subintervals, the following results hold $\left(t_{i} \triangleq i \Delta t\right)$.

Lemma 8. Consider $t_{0} \leq t_{k} \leq t_{l} \leq t_{n}$. Then

$$
\begin{gathered}
J_{o p t}^{m}\left(z_{0} ; t_{k}, t_{n}\right)=\min _{\sigma \in \Sigma^{m}} J_{\sigma}^{m}\left(z_{0} ; t_{k}, t_{n}, u_{\sigma^{o p t}\left(t_{k}, t_{n}\right)}^{o p t}\right) \\
=\min _{\sigma \in \Sigma^{m}}\left\langle z_{0}, \Pi_{\sigma\left(t_{k}, t_{n}\right)}\left(t_{k}\right) z_{0}\right\rangle \\
(1)=\left\langle z_{0}, \Pi_{\sigma^{o p t}\left(t_{k}, t_{n}\right)}\left(t_{k}\right) z_{0}\right\rangle . \\
\text { (2) } J_{o p t}^{m}\left(z_{0} ; t_{l}, t_{n}\right) \leq J_{o p t}^{m}\left(z_{0} ; t_{k}, t_{n}\right) \\
\text { (3) } \Pi_{\sigma^{o p t}\left(t_{l}, t_{n}\right)}\left(t_{l}\right) \leq \Pi_{\sigma^{o p t}\left(t_{k}, t_{n}\right)}\left(t_{k}\right) \\
\text { (4) }\left\|\Pi_{\sigma^{o p t}\left(t_{l}, t_{n}\right)}\left(t_{l}\right)\right\| \leq\left\|\Pi_{\sigma^{o p t}\left(t_{k}, t_{n}\right)}\left(t_{k}\right)\right\|
\end{gathered}
$$

It should be noted that the optimal switching function depends on the length of the interval $\Delta t$.

\section{TOWARDS OPTIMAL SWITCHING POLICY OF MA ON INFINITE-TIME INTERVAL}

In this section we consider the infinite-time interval with $t_{f}=\infty$. As a necessary condition for the solvability of Problem 1 on $\left[t_{0}, \infty\right)$, the existence of at least one input function $u$ and one switching function $\sigma$ that produce a finite cost $J_{\sigma}\left(z_{0} ; u\right)$ is required. This observation leads to the definition of optimizability of the family of switched systems.

Definition 9. The family of switched systems $\left(\left(S_{p}\right)_{p \in \mathcal{P}}, \Sigma\right)$ with the cost functional (4) is optimizable if at least one of the switched system $\left(\left(S_{p}\right)_{p \in \mathcal{P}}, \sigma\right)$ from the family is optimizable. This means that, for every $z_{0} \in Z$ there exist an input function $u \in L_{2}([0, \infty) ; U)$ and a $\sigma \in \Sigma$ such that the cost functional $J_{\sigma}\left(z_{0} ; u\right)$ is finite.

Note that, for every $p \in \mathcal{P}$, the definition for the optimizability of a subsystem $S_{p}$ which has a constant switching function $\sigma$ (no switch) corresponds to (Curtain and Zwart, 1995, Definition 6.2.1). If the whole state is accessible, i.e. $C_{p}=I$ for all $p \in \mathcal{P}$, then for each subsystem $S_{p}$ with a constant switching function, optimizability and exponential stabilizability are equivalent, see (Curtain and Zwart, 1995, Exercise 6.5). We also consider the following assumption.

Assumption 10. (Optimizability). Each of the subsystems $S_{p}$ of the family of linear continuous-time switched systems $\left(\left(S_{p}\right)_{p \in \mathcal{P}}, \Sigma\right)$ corresponding to constant switching functions $\sigma$ is optimizable.

Similar to the classical situation, the solution of the optimal control problem for switched systems is closely related to the control algebraic Riccati equations (CAREs)

$A^{*} \Pi+\Pi A-\Pi B_{p} B_{p}^{*} \Pi+C_{p}^{*} C_{p}=0$ on $D(A)$.

Assumption 10 is equivalent to the existence of solutions of the CAREs (13) for each $p \in \mathcal{P}$ (Curtain and Zwart, 1995, Exercise 6.9). Then the cost (4) on $\left[t_{f}, \infty\right)$, for $M=0\left(t_{0}\right.$ is replaced with $t_{f}$, and $t_{f}$ with $\left.\infty\right)$ is

$$
\begin{aligned}
& J_{o p t}\left(z_{0} ; t_{f}, u_{p}^{o p t}\right)= \\
& \int_{t_{f}}^{\infty}\left(\left\langle y_{p}^{o p t}(s), y_{p}^{o p t}(s)\right\rangle+\left\langle u_{p}^{o p t}(s), R u_{p}^{o p t}(s)\right\rangle\right) d s \\
&=\left\langle z_{p}\left(t_{f}\right), \Pi_{p} z_{p}\left(t_{f}\right)\right\rangle
\end{aligned}
$$

where $\Pi_{p}$ are solutions of CAREs (13). 


\subsection{Fixed number of switches}

Suppose that the family $\left(\left(S_{p}\right)_{p \in \mathcal{P}}, \Sigma\right)$ satisfies Assumptions 2-4 and Assumption 10. Moreover, consider that for a fixed switching interval $\Delta t$, a maximum of $n$ switches is allowed. Then the following algorithm provides the optimal switching policy for moving actuators with respect to the cost on the infinite-time interval.

Algorithm 2. Step 1: Take $t_{f} \triangleq n \Delta t$.

Step 2: For each $p \in \mathcal{P}^{m}$ find $M_{p}$, the smallest nonnegative solutions of the CARE (13).

Step 3: For each $p \in \mathcal{P}^{m}$, solve the optimal switching problem on the finite time interval $\left[t_{0}, t_{f}\right]$ with $M=M_{p}$. Using Algorithm 1, find the $m$ minimal costs of (11) as

$$
J_{p, o p t}^{m}\left(t_{0}, t_{f}\right)=\min _{\sigma \in \Sigma^{m}}\left\langle z_{0}, \Pi_{\sigma}^{p}\left(t_{0}\right) z_{0}\right\rangle
$$

Step 4: Find the minimal cost on $\left[t_{0}, t_{f}\right]$ as

$$
J_{o p t}^{m}\left(t_{0}, t_{f}\right)=\min _{p \in \mathcal{P}^{m}} J_{p, o p t}^{m}\left(t_{0}, t_{f}\right) .
$$

Step 5: Choose $\sigma^{\text {opt }}$ and $p_{\text {opt }}$ as the switching function and the index corresponding to the optimal cost $J_{o p t}^{m}\left(t_{0}, t_{f}\right)$. Take the optimal input $u_{\sigma^{o p t}}^{o p t}$ on $\left[t_{0}, \infty\right)$ as the one corresponding to $\sigma^{\text {opt }}$ and $p_{\text {opt }}$.

\subsection{Switching under stability assumptions}

Suppose that the family $\left(\left(S_{p}\right)_{p \in \mathcal{P}}, \Sigma\right)$ satisfies Assumptions 2-4 and Assumption 10. We replace the fixed number of switches assumption, taken in the previous subsection, with a stability assumption.

The closed-loop trajectory of any switched system on the finite-time interval $\left[t_{0}, t_{n}\right]$, satisfies

$$
z_{\sigma}\left(t_{n} ; z_{0}, t_{0}, t_{n}\right)=\prod_{i=0}^{n-1} U_{c l, \sigma\left(t_{i}\right)}\left(t_{i}, t_{i-1}\right) z_{0}
$$

where $U_{c l, p}(t, s)$ are the mild evolution operators on $\left\{(t, s) ; t_{i-1} \leq s \leq t \leq t_{i}\right\}$ with generators $A_{c l, p}(t)$ defined in (8). Then all switched systems are asymptotically stable (i.e. $z\left(t_{n}\right) \rightarrow 0$ as $t_{n} \rightarrow$ $\infty)$ if

$$
\prod_{i=0}^{n-1}\left(\gamma+\gamma^{2}+\gamma^{3} \frac{\left\|D_{\sigma\left(t_{i}\right)}\right\|^{2}(\Delta t)^{2}}{2}\right) \rightarrow 0
$$

as $n \rightarrow \infty$. Here $\gamma \triangleq \sup _{t \in[0, \Delta t]}\|T(t)\|$, and $D_{\sigma\left(t_{i}\right)} \triangleq-B_{\sigma\left(t_{i}\right)} R^{-1} B_{\sigma\left(t_{i}\right)}^{*} \Pi_{\sigma\left(t_{i}\right)}(t)$. We refer to (Curtain and Zwart, 1995, Proof of Theorem 3.2.5) for a bound of the mild evolution operator.

Assume that all switched systems from the given family are asymptotically stable. Then, for any $\epsilon>0$, there exists an $n \in \mathbb{N}$ such that

$$
\bar{M} \triangleq \max _{p \in \mathcal{P}^{m}, \sigma \in \Sigma^{m}}\left\langle z_{\sigma}\left(t_{n}\right), \Pi_{p} z_{\sigma}\left(t_{n}\right)\right\rangle<\epsilon .
$$

Then the following algorithm finds an $\epsilon$-optimal solution for Problem 5.

Algorithm 3. Step 1: Chose $\epsilon>0$ and $n=1$.

Step 2: For each $p \in \mathcal{P}^{m}$ find $M_{p}$, the smallest nonnegative solutions of the CARE (13).

Step 3: For any $\sigma \in \Sigma^{m}$ compute $z_{\sigma}\left(t_{n}\right)$, where $t_{n} \triangleq n \Delta t$.

Step 4: Calculate $\bar{M}$.

Step 5: If $\bar{M}>\epsilon$ take $n=n+1$. Return to Step 3 . Step 6: Continue with Step 3 in Algorithm 2.

The above algorithm can be further improved in order to reduce computational efficiency. One can replace $\bar{M}>\epsilon$ in Step 5 with a trade-off condition between the error on $\left[t_{0}, \infty\right)$ and a $\epsilon_{1}$ optimal condition on $\left[t_{0}, t_{f}\right]$.

\section{EXAMPLE}

The particular example in this paper considers operators $\left(B_{p}\right)_{p \in \mathcal{P}}$ which approximately model point actuators and sensors. When heating a metal rod (Curtain and Zwart, 1995, Example 4.1.2), one can consider $U=Y=\mathbb{C}, Z=L_{2}(0,1)$, and for each $p \in \mathcal{P}, B_{p} u=\frac{1}{2 \epsilon} \mathbf{1}_{\left[\xi_{0, p}-\epsilon, \xi_{0, p}+\epsilon\right]}(\xi) u$, where

$$
\mathbf{1}_{[\alpha, \beta]}=\left\{\begin{array}{ll}
1 & \text { for } \alpha \leq \xi \leq \beta \\
0 & \text { elsewhere }
\end{array} .\right.
$$

The system under examination is described by the following partial differential equation modelling metal heating and is given by

$\frac{\partial x}{\partial t}(t, \xi)=\frac{\partial}{\partial \xi}\left(\kappa(\xi) \frac{\partial x}{\partial \xi}(t, \xi)\right)+\frac{\mathbf{1}_{\left[\xi_{0, p}-\epsilon, \xi_{0, p}+\epsilon\right]}(\xi)}{2 \epsilon} u(t)$

where the spatially varying thermal parameter is

$$
\kappa(\xi)=0.01\left(1+0.25 \times \sin \left(\frac{3 \pi \xi}{L}\right) \times \sin ^{2}(\xi)\right) .
$$

Associated with the above PDE are the boundary conditions, taken here as Dirichlet with $x(t, 0)=$ $x(t, L)=0$ and $L=2$, and the initial conditions given by $x(0, \xi)=10 \sin (\pi \xi)$. It is assumed that the state is fully accessible, i.e. $C_{p}=I$ for every $p \in \mathcal{P}$. For this numerical study, we considered Algorithm 2 since we assumed that only three switches are allowed $(n=3)$. While Algorithm 2 considers the finite horizon case on the interval $\left[t_{0}, t_{f}\right]$, we also implemented Algorithm 3 in order to find the smallest final time $t_{f}$ that would satisfy (18). Once such final time is found, it is subsequently used in Step 1 of Algorithm 2 in conjunction with the fixed number of switches. The computations were carried out via codes written in Matlab ${ }^{\circledR}$ run on a dual processor DELL ${ }^{\circledR}$ workstation(Xeon $2.8 \mathrm{GHz}, 2 \times 2 \mathrm{~GB}$ ). The closed loop system was discretized using a spline-based Galerkin approximation scheme with 20 basis elements. The resulting finite dimensional system of ODEs was integrated using Matlab ${ }^{\circledR}$ ODE library. All required (spatial) integrals were computed 
numerically via a composite two point GaussLegendre quadrature rule. The resulting ODREs (9) were solved using the BDF 1-step method presented in (Benner and Mena, 2004). The set of candidate positions was chosen via

$$
\mathcal{P}^{m}=\left\{p_{j}: p_{j}=\frac{(j-0.137) L}{m+1}, j=1, \ldots, m\right\},
$$

with $m=3$ and the LQR parameters were chosen as $R=1, Q=4 \times 10^{-2} I, M=I$. Using Algorithm 3 , the final time $t_{f}$ for the $\epsilon$-optimal solution to Problem 5 with a level $\epsilon=0.8$ in (18) resulted in $t_{f}=21$ with $\Delta t=7 \mathrm{sec}$. With that value of $t_{f}$, Algorithm 2 was implemented for the finite horizon $[0,21]$. The system was then simulated for the interval $[21,35]$ using a controller based on the infinite-horizon problem. Specifically, the optimal actuator switching scheme predicted the sequence 331 meaning that for the interval $[0,7]$ the $3^{\text {rd }}$ actuator was used, for the time interval $[7,14]$ the $3^{\text {rd }}$ actuator was used again, and for the time interval $[14,21]$ the $1^{\text {st }}$ actuator was employed. The controller then employed actuator \#1 for the interval $[21,35]$ using a constant feedback gain obtained from the solution to the associated CARE in (13). As a comparison, a non-switching actuator was considered which employed the $1^{\text {st }}$ actuator throughout the finite horizon $[0,21]$ that used the optimal control (7) via the solution to (9). Continuing, the infinite horizon problem was solved for $[21,35]$ using the $1^{\text {st }}$ actuator. The time evolution of the $L_{2}$ norm of the closed loop system is presented in Figure 1, where the optimal switching case is depicted by a solid line and the (nonswitching) optimal case with a fixed actuator is depicted by a dashed line. One can easily observe the performance improvement when both the actuator and the control signal are allowed to switch. The associated actuator switching sequence for both cases is presented in Figure 2 .

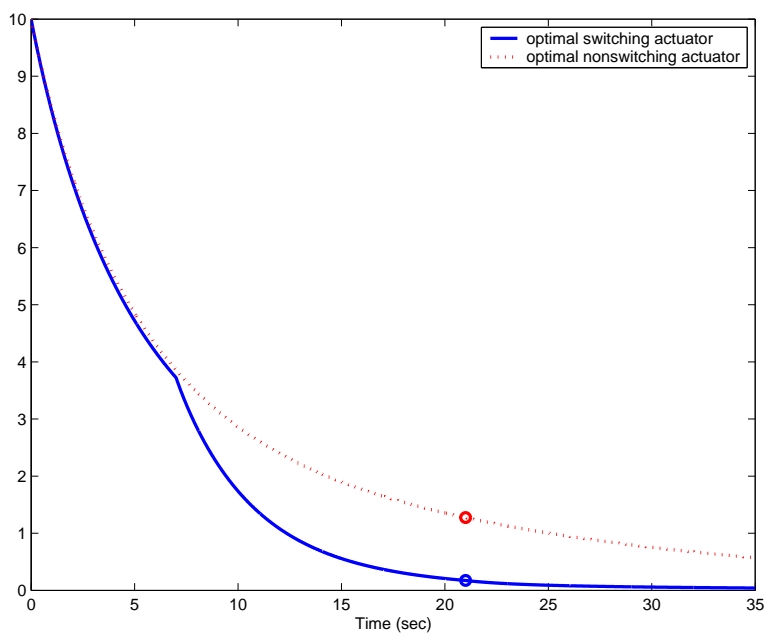

Fig. 1. $L_{2}(0, L)$ norm of $x(t, \xi)$ vs time; switching actuator (solid) and fixed actuator (dashed).

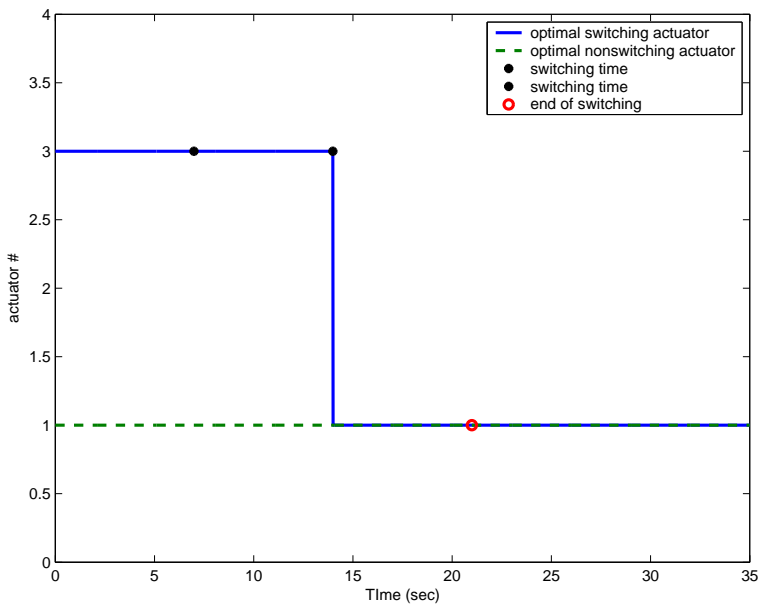

Fig. 2. Actuator allocation (switching) for $m=3$ actuator locations; switching (solid) and fixed (dashed).

\section{CONCLUSIONS}

We have presented an algorithm that allows for the optimal actuator and controller switching policy in a class of hybrid DPS. In addition to providing the theoretical results for the finite horizon case, two additional cases were considered which account for: a fixed number of switches and for the case of choosing the minimum finite horizon limit. Beyond these cases the system no longer required any switching. The latter case allows one to employ stability assumptions to find the smallest limit of the finite horizon optimal control problem. Extensive simulation studies combining various algorithms were presented and which exhibited the superior performance of the hybrid system when an actuator was allowed to switch.

\section{REFERENCES}

Benner, P. and H. Mena (2004). BDF methods for large-scale dfifferential Riccati equations. In: Proc. MTNS. Leuven, Belgium.

Borrelli, Francesco (2003). Constrained Optimal Control of Linear and Hybrid Systems. Lecture Notes in Control and Information Sciences, vol. 290. Springer-Verlag. New York.

Butkovskiy, A. G. and L. M. Pustyl'Nikov (1987). Mobile Control of Distributed Parameter Systems. Ellis Horwood Limited. Chichester.

Curtain, R. F. and H. J. Zwart (1995). An Introduction to Infinite Dimensional Linear Systems Theory. Texts in Applied Mathematics, vol. 21. Springer-Verlag. New York.

Demetriou, M. A., A. Paskaleva, O. Vayena and H. Doumanidis (2003). Scanning actuator guidance scheme in a 1-D thermal manufacturing process. IEEE Tr. on Contr. Sys. Tech. 11(5), 757-764.

Liberzon, Daniel (2003). Switching in Systems and Control. Birkhäuser. Boston.

Savkin, Andrey V. and Robin J. Evans (2002). Hybrid Dynamical Systems. Birkhauser. Boston. 